\title{
The Influence of Temperature on Streptomycin Inhibition Zones in Agar Gultures
}

\author{
By K. E. COOPER And W. A. GILLESPIE \\ Bacteriology Laboratories, Bristol University, and Bristol Royal Infirmary
}

\begin{abstract}
SUMMARY: The diffusion of streptomycin in agar gel seeded with staphylococci as in assay methods, results in a clearly defined zone of inhibition. The time required for this definition to develop is about $2 \frac{1}{2} \mathrm{hr}$. at $37^{\circ}$, but varies greatly with temperature and is equal to the lag period plus approximately 4 times the generation time. After 4-5 generations the young colonies become much less susceptible and grow unimpeded by the rapidly increasing concentration of the antibiotic reaching them. Incubation at temperatures below $37^{\circ}$, although without significant effect on the minimum inhibitory concentration, results in larger inhibition zones because of increased generation times.

In assays and sensitivity tests by diffusion methods, errors will occur if organisms under comparison do not multiply at the same rate.
\end{abstract}

When an antiseptic diffuses into nutrient agar seeded with a sensitive organism (as in the standard diffusion assay methods) there is a race between the drug diffusing into the medium, and the growth of the colonies. At first sight it might seem that the edge of the zone of inhibition occurs at a point where colonies have grown to macroscopically visible size or to full size before the minimum inhibitory concentration of the antiseptic reaches them. This explanation, however, does not account for the sharpness of the edge of the inhibited zone seen with some antibiotics. Experiments with streptomycin indicate that when the young colonies have grown for a certain critical, and quite short, time, they become much less susceptible to the action of streptomycin, and that they continue to grow to full and even to enhanced size in spite of the presence around them of much more than the minimum inhibitory concentration. Thus the edge of the zone is defined before the colonies become visible to the naked eye.

The experiments with streptomycin using the Mayo strain of staphylococcus were concerned in the first place with the effect of temperature on the size of the inhibited zone. The formula which we used was suggested by Cooper \& Woodman (1946). This formula relates the distance travelled by the minimum inhibitory concentration of antiseptic in any particular time to the concentration of the antiseptic placed in the cup or on top of the column of agar. It rests on theoretical principles which apply to the tube assay method for streptomycin (described by Mitchison \& Spicer, 1949) where the agar seeded with the organism is placed in narrow tubes, and the streptomycin is placed on top of the agar column. After incubation the length of the resulting zone of inhibition is measured with a microscope vernier. In the equation of Cooper \& Woodman (1946): $\quad x^{2}=4(2 \cdot 30 D T)\left(\log m_{0}-\log m^{\prime}\right)$;

$x=$ length $\left(\mathrm{mm}\right.$.) of inhibited zone, $m_{0}=$ concentration of antiseptic placed GM VII I $\$ 2$ 
in contact with the agar, $m^{\prime}=$ minimum inhibiting concentration of the antiseptic in agar, before a critical time (called here $T_{0}$ ) after the start of growth, $\boldsymbol{T}=$ time (hr.) the antiseptic has been diffusing, and $D=$ diffusion coefficient (mm. ${ }^{2} / \mathrm{hr}$.).

In the usual method of assay, antibiotic is added to the tubes at the moment when they are warmed to the incubation temperature. Diffusion and growth begin at the same moment, and we propose to call the particular value of $T$ which must be used to fit the theoretical line to the experimental points $T_{0}$. Most workers have not determined this constant because, since $D$ is also constant in a particular experiment, $D T_{0}$ is the only constant in addition to $m^{\prime}$ which must be known in order to draw the theoretical curve. This product $D T_{0}$ determines the slope of the normal assay line.

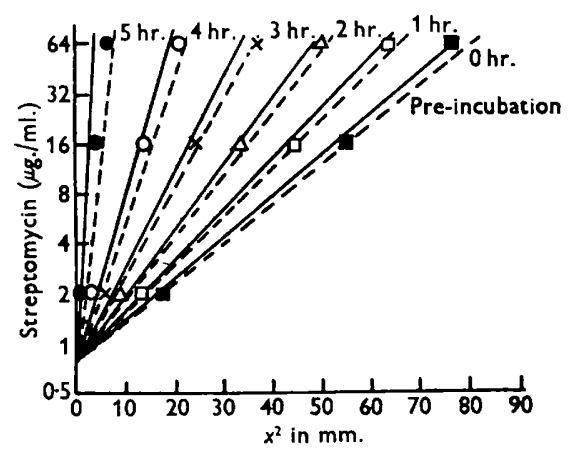

Fig. 1

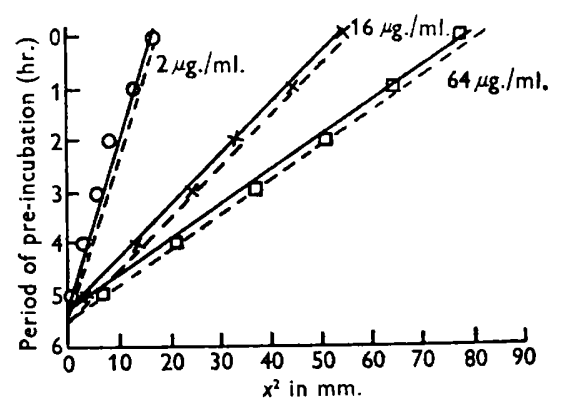

Fig. 2

Fig. 1. Effect of pre-incubation on zones of inhibition of staphylococcus by streptomycin at $27^{\circ}$. Concentration on a logarithmic scale is plotted against $x^{2}$, where $x=$ depth of inhibited zone. The hour of pre-incubation represents the length of time before streptomycin was added to the tubes, for each set of determinations. Theoretical lines for $T_{0}=5 \cdot 5,---$, and $T_{0}=5 \cdot 25, \longrightarrow$, are given when $D=0.84 \mathrm{~mm} .{ }^{2} / \mathrm{hr}$. and $m^{\prime}=0.75 \mu \mathrm{g} . /$ ml. Period of pre-incubation (hr.): $0, \square ; 1, \square ; 2, \triangle ; 3, \times ; 4,0 ; 5,0$.

Fig. 2. Graph giving the same results as Fig. 1 but showing the constancy of $T_{0}$ for different concentrations of streptomycin. $D=0.84 \mathrm{~mm} .^{2} / \mathrm{hr} . m^{\prime}=0.75 \mu \mathrm{g} . / \mathrm{ml}$. Theoretical lines $T_{0}=5 \cdot 5,--, T_{0}=5 \cdot 25,-$ Experimental points, streptomycin $\mu$ g. $/ \mathrm{ml} .: 64, \square$; $16, x ; 2,0$.

Other assays can be made with a different value of $T$ by pre-incubating the tubes before adding the antibiotic. The experiments of Schmidt \& Moyer (1944), as was explained by Cooper \& Woodman (1946), were of this type. If incubation and hence growth proceeds for $h \mathrm{hr}$. before the antibiotic is added, then growth will have proceeded for $T_{0} \mathrm{hr}$. as soon as $T_{0}-h \mathrm{hr}$. have elapsed since diffusion started. The assay line which results will be given by using $T_{0}-h$, instead of $T$ in the formulae.

A series of such lines is shown in Fig. 1, which records experiments carried out at $27^{\circ}$. When $h$ was increased until it became greater than $T_{0}$, no inhibition occurred no matter how much streptomycin was added to the tubes.

These results can be shown in another way by plotting $h$ against $x^{2}$ for each concentration $m_{0}$ used:

$$
h=T_{0}-\frac{x^{2}}{9 \cdot 2 D\left(\log m_{0}-\log m^{\prime}\right)}=T_{0}-\frac{x^{2}}{K} .
$$


This is shown in Fig. 2 and gives also a straight line for each concentration provided no change in $m_{0}$ or $m^{\prime}$ occurs in the time $T_{0}$. When $h=T_{0}$ then $x^{2}=0$. The method of determining $T_{0}$, then, is to find where the $x^{2} / h$ graph cuts the $h$ axis, and if the theory is correct this point should be independent of concentration. It will be seen that almost all the points lie between lines for $T_{0}=5 \cdot 5$ and $T_{0}=5 \cdot 25 \mathrm{hr}$.

To obtain this agreement care had to be taken over many technical details, particularly those concerned with temperature control and time. The rapid inoculation of liquid agar at a temperature of $45^{\circ}$, its setting and cooling for a standard time, its placing in the water-bath at the required temperature and the use of antibiotic solution previously warmed to this temperature are examples. Other details are described in the experimental section of the paper.

When corresponding graphs at $37^{\circ}$ were plotted the minimum inhibitory concentration $m^{\prime}$ remained unchanged at 0.75 units, but $T_{0}$ was considerably less, namely $2.75 \mathrm{hr}$. It is indeed remarkable that at this temperature, in as short a time as $3 \mathrm{hr}$., the colonies of staphylococci became insusceptible to high concentrations of streptomycin, though the individual organisms on subculture appear to be as sensitive as ever.

It is not proposed to show sets of graphs for each temperature investigated, but the effect of temperature on the normal assay curves when $h=0$ (diffusion and growth starting at the same time), is shown in Fig. 3. Theoretical curves have been drawn from the formula with the concentration $m^{\prime}=1$ because, although variations occurred in individual experiments from 0.75 to $1 \cdot 25$, we decided that their importance was doubtful, and that the total results were best expressed by using this mean value. Secondly, we give in Fig. 4 the calculated $h / x^{2}$ lines for a concentration of $64 \mu \mathrm{g}$. streptomycin $/ \mathrm{ml}$. as this shows the very great effect of temperature on $T_{0}$ which increases from $2 \cdot 25$ at $42^{\circ}$ to $9 \cdot 0$ at $22^{\circ}$. Both figures show close agreement with the experimental points. The lower temperatures give much larger zones of inhibition.

It will be noticed that in Fig. 4 the lines are not parallel but slightly converging. This convergence is due to the effect of temperature on $D$, which can be calculated from the effect of temperature upon the viscosity of water. The dependence of $D$ on absolute temperature and viscosity was described in the paper by Cooper \& Woodman (1946). The values for the results at $42^{\circ}$, calculated in this way, agree very well with the observed values. Table 1 shows the values of the constants $D, T_{0}$ and $m^{\prime}$ which best fit the whole series of points obtained by experiments conducted at each temperature for different concentrations and at varying periods of pre-incubation. The changes in $D$ and $T_{0}$ with temperature are in opposite directions and, though the effect of $T_{0}$ is much the greater, the product $D T_{0}$ gives a minimum value at $37^{\circ}$. The results at $42^{\circ}$ are very similar to those at $37^{\circ}$, though findings at lower temperatures differ greatly. The calculated relative values for $D$ are given in Table 1 and show good agreement.

It was then noticed that the effect of temperature on the generation times of a number of organisms-Bacterium coli, streptococci, etc., reported in the 
literature were proportional approximately to our $T_{0}$ values. As no record could be found for the staphylococcus, determinations were made of the generation time of the strain used in the broth base of our agar medium, seeded in the same way with the same number of organisms.

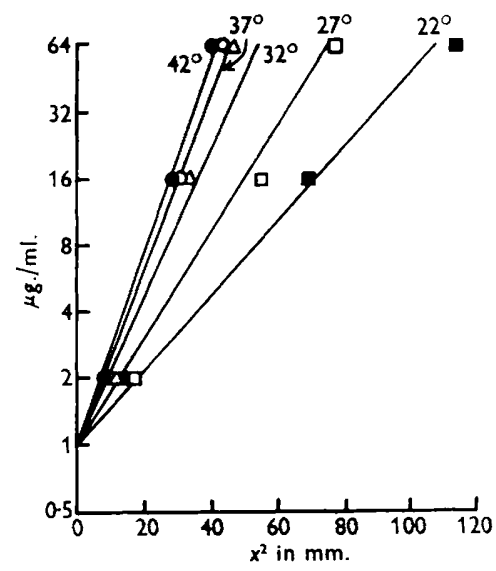

Fig. 3

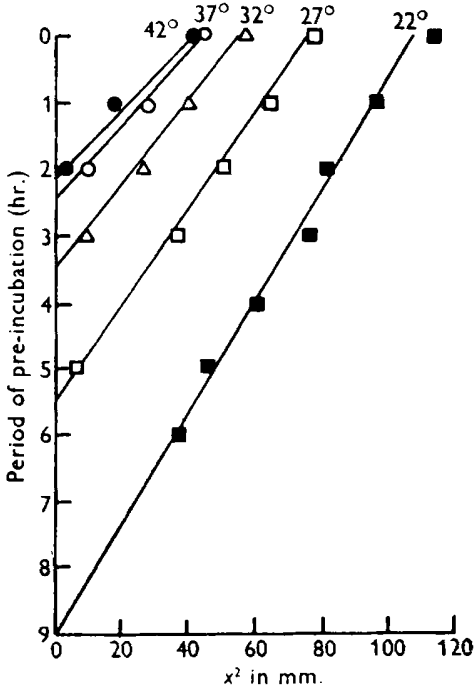

Fig. 4

Fig. 3. Streptomycin inhibition zone with staphylococcus in tubes. The relation of $x^{2}$ to $\log$ concentration for different temperatures, without pre-incubation. Theoretical lines are drawn with $m^{\prime}$ independent of temperature and values of $D$ calculated from the values of the viscosity of water for each temperature (see Table 1). $h=0 ; m^{\prime}=1$. Experimental points: $O, 42^{\circ} ; O, 37^{\circ} ; \triangle, 32^{\circ} ; \square, 27^{\circ} ; \mathbf{\square}, 22^{\circ}$.

Fig. 4. Streptomycin inhibition zones with staphylococcus in tubes, at different temperatures. The influence of temperature on $T_{0}$ is shown by the results of pre-incubation with one concentration of streptomycin $(64 \mu \mathrm{g} . / \mathrm{ml}$.). The theoretical lines use the calculated relative values of $D$ for the different temperatures and a constant value for the minimum inhibitory concentration $m^{\prime}=1$. Experimental points : $0,42^{\circ} ; \bigcirc, 37^{\circ} ; \triangle, 32^{\circ} ; \square, 27^{\prime \prime}$; 口, $22^{\circ}$.

Table 1. Influence of temperature on constants of assay curves Streptomycin on staphylococcus (Mayo)

$\begin{array}{cccccc}\text { Temp. } & D & D & T_{0} & \\ \left({ }^{\circ}\right) & \text { observed } & \text { calculated } & \text { observed } & D T_{0} & m^{\prime} \\ 42 & 1 \cdot 15 & 1 \cdot 15 & 2 \cdot 2 & 2 \cdot 53 & 1 \cdot 00 \\ 37 & 0 \cdot 92 & 1 \cdot 03 & 2 \cdot 5 & 2 \cdot 30 & 0 \cdot 75 \\ 32 & 0 \cdot 89 & 0.92 & 3 \cdot 5 & 3 \cdot 12 & 0 \cdot 90 \\ 27 & 0 \cdot 84 & 0 \cdot 82 & 5 \cdot 5 & 4 \cdot 63 & 0 \cdot 75 \\ 22 & 0 \cdot 75 & 0.72 & 9 \cdot 0 & 6 \cdot 85 & 1 \cdot 25\end{array}$

Results in tubes in water-baths at controlled temperature.

Table 2 shows that the ratio of $T_{0}$ to the generation time is approximately 5.5 over the range of active growth from 22 to $42^{\circ}$. A still more constant ratio is obtained by allowing for the lag period which was experimentally determined, a correction which reduces it to 4 . In other words, the critical change 
in susceptibility from colonies inhibited by $\mathrm{m}^{\prime}$, to colonies insusceptible to considerably higher concentrations of streptomycin occurs between the development of the 4 th and 5 th generations of descendants of the original inoculum.

Table 2. Influence of temperature on generation time and lag period

\begin{tabular}{|c|c|c|c|c|c|}
\hline \multicolumn{6}{|c|}{ Streptomycin on staphylococcus (Mayo) } \\
\hline $\begin{array}{c}\text { Temp. } \\
\left({ }^{\circ}\right)\end{array}$ & $\begin{array}{c}T_{0} \\
\text { (hr.) }\end{array}$ & $\begin{array}{c}\text { G.T. } \\
\text { (min.) }\end{array}$ & $\begin{array}{c}\text { L. } \\
\text { (hr.) }\end{array}$ & $T_{0} / \mathrm{G.T}$ & $T_{0}-\mathbf{L} . /$ G.T. \\
\hline 42 & $\mathbf{2 \cdot 2}$ & $25 ?$ & $0 ?-0.5 ?$ & $5 \cdot 3$ & $5 \cdot 3-4 \cdot 1$ \\
\hline 37 & $2 \cdot 5$ & 29 & $0 \cdot 8$ & $5 \cdot 0$ & $\mathbf{3 \cdot 5}$ \\
\hline 32 & $3 \cdot 5$ & 29 & $1 \cdot 0$ & $5 \cdot 4$ & $\mathbf{3 \cdot 9}$ \\
\hline 27 & $5 \cdot 5$ & 54 & 1.5 & $6 \cdot 1$ & $4 \cdot 4$ \\
\hline \multirow[t]{2}{*}{22} & $9 \cdot 0$ & 86 & $3 \cdot 0$ & $6 \cdot 3$ & $4 \cdot 2$ \\
\hline & & & Av & $5 \cdot 6$ & $4 \cdot 1$ \\
\hline
\end{tabular}

Results in tubes in water-baths at controlled temperature. G.T.=generation time; L. = lag period.

\section{DISCUSSION}

The main effect of temperature on the zone of inhibition has been shown to be due to the changed rate of growth of the organism. Effects on minimum inhibitory concentration $\left(m^{\prime}\right)$ and on the diffusion coefficient $D$ are of much less importance. $T_{0}$ has been found to be given approximately by $T_{0}=4 \cdot 1$ (generation time) +lag period. The value of the minimum inhibitory concentration of streptomycin in agar for the Staphylococcus aureus strain Mayo is little affected by temperature. Nevertheless, the size of the inhibited zones of growth is greatly affected because of the large variation in $T_{0}$ (and a small variation in $D)$. Between 22 and $42^{\circ}$ the formula $x^{2}=4(2 \cdot 30 D)\left(T_{0}-h\right)$ $\left(\log m_{0}-\log m^{\prime}\right)$ agrees within the limits of experimental error with the facts investigated, and there seems to be no advantage in using more complicated formulae. The theoretical significance of the resistance of the colony which arises after four generations (with the strength of inoculum used in these experiments) will be discussed elsewhere. It will be of interest to examine other organisms and other antiseptics by these methods. We suspect from the varying nature of the boundary zone between inhibition and growth with different antiseptics, that factors not yet considered may affect the zone. The rate of action of the antiseptic at concentrations near $m^{\prime}$ has not been considered in the simplified theory proposed, which assumed that concentrations of $m^{\prime}$ and above inhibited instantly growth up to time $T_{0}$, but were without effect after time $T_{0}$. It has also been assumed that concentrations below $m^{\prime}$ are without any effect. These are obviously only partially correct assumptions, and it is not surprising therefore that zones obtained with other organisms and antibiotics may not always be as easy to measure, or agree so well with the theory as results obtained with streptomycin and staphylococcus.

Apart from the theoretical implications of these facts, there are some practical conclusions to be drawn from these experiments. One is that when antibiotic assays are done by the agar-diffusion methods, care must be taken 
to ensure that the temperature of the medium is exactly the same in all the tubes and plates used. For this reason, tubes are in general better than plates, and a water-bath than an incubator. There is also a practical bearing on sensitivity tests done by the agar-diffusion method (as in a ditch or cup plate). If two organisms which are being compared on the same plate have significantly different generation times, conclusions about sensitivity may be erroneous. Such apparent differences in sensitivity might arise if the medium used were relatively more suitable for one of the organisms.

\section{EXPERIMENTAL DETAILS}

Determination of inhibition zones. The assay method of Mitchison \& Spicer (1949) was used, with some modifications.

Medium. Equal parts of nutrient (infusion) agar and a $1 \%$ solution of peptone (Evans), adjusted to $\mathrm{pH} \mathbf{7} \cdot 8$ and stored in $50 \mathrm{ml}$. quantities.

Test organism. The 'Mayo' strain of Staph. aureus.

Agar diffusion tubes. Narrow tubes (3 mm. internal diameter) as used for assays.

Method of incubation. Accurately controlled water-baths which could be adjusted between 22 and $42^{\circ}$ were used.

Procedure. The object of the experiments was to obtain a set of streptomycin standard assay curves at each temperature, by varying the length of time for which growth was allowed to occur before the streptomycin solutions were placed in contact with the columns of seeded agar. Thus there were obtained, at each temperature, curves for $0,1,2,3$, etc., hr. of pre-incubation. The temperatures used were $22,27,32,37$ and $42^{\circ}$. Two such sets of curves were obtained in each experiment, one at $37^{\circ}$ and one at another of the temperatures. The $37^{\circ}$ results thus served as controls for the comparisons of curves obtained on different occasions. Particular attention was given to the exact timing of periods of pre-incubation, accurate temperature control and uniform treatment of the test organism.

Three standard solutions of streptomycin in distilled water were used in constructing the curves, their concentrations being 2,16 and $64 \mu \mathrm{g}$. streptomycin base $/ \mathrm{ml}$. These were made up at the beginning of each experiment from a stock $10,000 \mu \mathrm{g} . / \mathrm{ml}$. solution kept at $4^{\circ}$, and were warmed in the water-bath before adding to the assay tubes. For each standard solution, three assay tubes were used, so that to obtain a single curve a row of nine tubes was required. One such row was put up for each period of pre-incubation.

To set up an experiment, one bottle of medium was melted and its contents distributed in $9 \cdot 5 \mathrm{ml}$. amounts in $1 \mathrm{oz}$. screw-capped McCartney bottles which were placed in a $45^{\circ}$ water-bath. Sufficient time was allowed for temperature equalization. Two-tenths ml. of an overnight $\left(37^{\circ}\right)$ infusion broth culture of the staphylococcus was added to $10 \mathrm{ml}$. sterile distilled water at $37^{\circ}$, mixed and kept in the incubator. Five-tenths ml. of the diluted culture was added to 1 bottle of melted medium, which was thoroughly mixed, replaced in the $45^{\circ}$ water-bath and used to fill the eighteen sterile assay tubes comprising two rows (one to be incubated at $37^{\circ}$ and one at the other temperature). After 
filling, the tubes were not re-plugged. The times of commencing and completing the filling of each row were noted. The tubes were fixed by means of plasticine vertically on the bench (at 18 to $22^{\circ}$ ) for $15 \mathrm{~min}$. (During this period another bottle of medium was seeded and filled into tubes.) The tubes were then placed in Wassermann racks in the appropriate water-baths. The warmed streptomycin solutions were added quickly, either at once or after the planned periods of pre-incubation, by means of fine Pasteur pipettes and without removing the assay tubes completely from the bath. After incubation overnight, the zones of inhibition were measured by means of a microscope vernier scale.

Determination of generation time. The medium was the same as that used for the determination of inhibition zones, except that infusion broth replaced the nutrient agar. An inoculum of the same size was used and the seeded medium held first at $45^{\circ}$ and then on the bench for the same periods as when determining inhibition zones before incubating in the water-bath at the required temperature. Viable counts (Miles \& Misra, 1938) were done at hourly intervals. The results were plotted and the lag period and generation time were determined from the intersection and slope of the best straight line with the horizontal through the level of the initial count.

We should like to express our thanks to Mrs N. Webster for technical assistance.

\section{REFERENCES}

Cooper, K. E. \& Woodman, D. (1946). The diffusion of antisepties through agar gels, with special reference to the agar cup assay method of estimating the activity of penicillin. J. Path. Bact. 58, 75.

Mires, A. A. \& Misra, S. S. (1938). The estimation of the bactericidal power of blood. J. Hyg., Camb. 38, 732.

Mrtchison, D. A. \& Spicer, C. C. (1949). A method of estimating streptomycin in serum and other body fluids by diffusion through agar enclosed in glass tubes. J. gen. Microbiol. 3, 184.

Schmidt, W. H. \& Moyer, A. J. (1944). Penicillin. I. Methods of assay. J. Bact.47, 199. 